\title{
Patolojik Dinlenme Tremorlerinin Kompleks Düzlemde Adaptif Tahmini
}

\author{
Buket ÇOLAK GÜVENÇ${ }^{1 *}$, Engin Cemal MENGÜÇ \\ 1* Kayseri Üniversitesi, Mühendislik, Mimarlık ve Tasarım Fakültesi, Elektrik-Elektronik Mühendisliği Bölümü, Kayseri, Türkiye, (ORCID: 0000-0003-0805-5885), \\ buketguvenc@kayseri.edu.tr \\ ${ }^{2}$ Kayseri Üniversitesi, Mühendislik, Mimarlık ve Tasarım Fakültesi, Elektrik-Elektronik Mühendisliği Bölümü, Kayseri, Türkiye, (ORCID: 0000-0002-0619-549X), \\ enginmenguc@kayseri.edu.tr
}

(International Conference on Design, Research and Development (RDCONF) 2021 - 15-18 December 2021)

(DOI: 10.31590/ejosat.1039914)

ATIF/REFERENCE: Çolak Güvenç, B. \& Mengüç, E. C. (2021). Patolojik Dinlenme Tremorlerinin Kompleks Düzlemde Adaptif Tahmini. Avrupa Bilim ve Teknoloji Dergisi, (32), 318-325.

Öz

$\mathrm{Bu}$ çalışmada, Parkinson hastalarında sıklıkla görülen patolojik dinlenme tremorlerinin kompleks düzlemde adaptif tahmini gerçekleştirilmiştir. Bu kapsamda, ilk olarak anlık olarak ölçülen patolojik "sağ el ve sol el" veya "sağ bacak ve sol bacak" tremorleri kompleks düzlemde ifade edilmiş ve ardından, bu kompleks-değerli patolojik tremorler, bir-adım-ileri kesin linear (strcitly linear, SL) ve geniş linear (Widely linear, WL) tabanlı tahmin ediciler vasıtasıyla adaptif olarak tahmin edilmiştir. Burada, SL tabanlı tahmin edici, kompleks-değerli en küçük ortalama kare (Complex-valued least mean square, CLMS) algoritması ile eğitilirken, WL tabanlı tahmin edici ise artırılmış CLMS (Augmented CLMS) algoritması ile eğitilmiştir. Tahmin edicilerin başarımları, gerçek dünya verisi olan patolojik dinlenme tremorleri üzerinde mutlak hata ve tahmin kazancı açısından incelenmiştir. Yapılan benzetim sonuçları; kompleksdeğerli patolojik dinlenme tremorlerinin dairesel olmayan davranış sergilediğini ve bu yüzden de WL tabanlı tahmin edicinin, SL versiyonuna kıyasla daha üstün bir başarım sergilediğini ortaya koymuştur.

\section{Adaptive Prediction of Pathological Resting Tremors in the Complex Domain}

\begin{abstract}
In this study, adaptive estimation of pathological resting tremors, which is frequently encountered in Parkinson's patients, is performed in the complex domain. In this context, pathological "right hand and left hand" or "right leg and left leg" tremors, which were measured instantaneously, are first expressed in the complex domain. Then, these complex-valued pathological tremors are predicted adaptively using one-step-ahead strictly linear (SL) and widely linear (WL) based predictors. Here, the SL based predictor is trained by the Complex-valued least mean square (CLMS) algorithm, while the WL based predictor is trained by the augmented CLMS (ACLMS) algorithm. The performances of these predictors were examined in terms of absolute error and prediction gain on pathological resting tremors as real-world data. Simulation results reveal that complex-valued pathological resting tremors exhibit non-circular behavior and thus the WL based predictor outperforms the SL version.
\end{abstract}

Keywords: Parkinson's disease, Resting Tremor, Complex Domain, Adaptive Prediction.

\footnotetext{
*Sorumlu Yazar: buketguvenc@kayseri.edu.tr
} 


\section{Giriş}

Bir biyomedikal sinyal tipi olan tremor, kasların ritmik olarak kasılması sonucu ortaya çıkan istem dışı bir hareket olarak tanımlanır (Elble ve Koller, 1990). Bu tip sinyaller, karakteristiksel olarak sinüsoidal sinyallere oldukça benzerdir ve insan vücudunda; el bilekleri ve dirsek eklemlerinde oluşur (Elble ve Koller, 1990). Tremor sinyalleri genel anlamda fizyolojik tremor ve patolojik tremor olarak iki sınıfa ayrılır. Fizyolojik tremor, yüksek frekanslı ve düşük genliklikli bir sinyal olup, literatürde bu tremor çeşidine insanların çoğunda rastlandığını gösteren klinik çalışmalar mevcuttur (Riviere vd., 2003). Patolojik tremor ise 3-14 Hz arasında bir frekans bandına sahiptir ve sinir sistemi hastalıkları kaynaklıdır (Elble, 1997; Wang vd., 2014). İnsan yaşamını tehdit edici olmayan patolojik tremorler; Parkinson, esansiyel tremor ve çoklu skleroz hastalarında görülmektedir. Fakat istemsiz salınımlarından dolayı hastalarda; günlük yaşamın kalitesini azaltmakta, hedefleme ve koordinasyon bozukluğu gibi kısıtlayıcı durumlara sebep olmaktadır (Riviere ve Thakor, 1996; Elble, 1997; Wang vd., 2014). Bu tür hastaların kullanabileceği giyilebilir, yardımcı ve iyileştirici yeni teknolojilerin geliştirilebilmesi için tremor sinyallerinin minimum gecikme ile yüksek doğrulukta anlık olarak modellenmesi veyahut tahmin edilmesi ciddi anlamda önem arz etmektedir (Atashzar vd., 2016; Rocon vd., 2007a; Rocon vd., 2007b; Meneski, 2011; O’Connor ve Kini, 2011; Kiguchi ve Hayashi, 2012).

$\mathrm{Bu}$ amaç doğrultusunda, literatürde adaptif Fourier lineer birleştirici (Fourier Linear Combiner, FLC), ağırlıklandırılmış frekans (weighted) FLC (WFLC) ve bant sinırl FLC yapıları geliştirilmiştir (Riviere ve Thakor, 1996; Atashzar vd., 2016; Vaz vd., 1994; Riviere vd., 1997; Riviere vd., 1998; Veluvolu vd., 2007; Veluvolu and Ang, 2010; Veluvolu vd., 2010; Rezayi ve Mengüç, 2018, Mengüç vd., 2021). Bu yapılar, genellikle istemli hareketin mevcut olduğu tremorlerin modellenmesinde kullanılan etkin yapılar olarak literatürde yer almaktadır. Son zamanlarda, yapılan bir çalışmada, WFLC yapısının modelleme başarımını dinlenme tremorleri üzerinde test etmiştir (Rezayi ve Mengüç, 2019). Literatürde bugüne kadar yapılan çalışmalar incelendiğinde; önerilen yapıların sadece modelleme ve kestirim amaçlı kullanıldığı ve bu yüzden geçmiş verileri dikkate almadan anlık veriler ile bu görevleri yerine getirtiği açıkça görülmektedir. Ayrıca, bu yapılar reel düzlemde çalıştığından tek kanallı tremor ölçümleri için uygundur. Aynı anda iki kanaldan alınan tremor sinyallerinin yukarıda bahsedilen yapilarla modellenebilmesi veya kestirilebilmesi için aynı özelliklere sahip iki yapının paralel olarak çalıştırılması gerekmektedir. Ancak, bu tip paralel yapılar, kanallar arasındaki ilişkiyi ne yazık ki dikkate almamaktadır. Sonuç olarak; yapılan çalışmalarda, adaptif filtrelerin sinyal tahmin yeteneğinin ve her iki kanalı dikkate alan kompleks düzlemdeki çalışma özelliğinin kullanılmaması literatürde hala eksiktir.

Günümüzde sensör teknolojisindeki son gelişmeler, artan bilgisayar bellekleri ve hesaplama gücünün artışı gösteriyor ki sinyal işleme alanında yalnızca reel-değerli sinyallerin işlenmesi ile uğraşılmamakta; aynı zamanda kompleks-değerli sinyallerin de işlenmesi gerekmektedir (Stott vd., 2018). Fiziksel olarak kıyaslandığında kompleks-değerli sinyaller, reel-değerli sinyallere göre, daha anlamlı bir analiz için firsat yaratan zengin bir yapıya sahiptir. Kompleks-değerli sinyaller ile doğası gereği, haberleşme, sinyal işleme, güç sistemleri ve biyomedikal gibi birçok farklı alanda karşılaşılmaktadır. Kompleks-değerli sinyaller, hem genlik hem de faz bilgisini doğal olarak yapısında bulunduran sinyal tipleri olup, "reel ve imajiner" veya "faz ve genlik" olarak iki farklı şekilde tanımlanabilmektedir. Ayrıca, literatürde kompleks-değerli sinyal işlemenin gerekli olduğu çok farklı durumlar da mevcuttur (Mandic ve Goh, 2009; Adali ve Haykin, 2010). Örneğin; bir eş-fazlı ve bir karesel bileşenden oluşan doğal kompleks forma sahip ve bu iki bileşen arasındaki ilişkiyi tamamen hesaba katan doğal kompleks-değerli sinyallerin işlenmesi gerekir. $\mathrm{Bu}$ tip sinyallere, radar, MRI (Adali ve Calhoun, 2007), ikili faz kaymalı anahtarlama (BPSK), kareleme faz kaymalı anahtarlama (QPSK) ve kareleme genlik modülasyonu (QAM) gibi birçok iletişim sinyali örnek verebilir. Öte yandan, harmonik analiz, Fourier analizi, kompleks dalgacık dönüşümü (complex wavelet transform) sinyal işleme için sıklıkla kullanılan araçlardır ve bu tip dönüşüm işlemlerinin gerçekleştirilmesi gereken durumlarda, kompleks-değerli sinyallerin işlenmesi gerekmektedir (Adali ve Calhoun, 2007). Bunlara ek olarak, kompleks düzlem, "genlik ile faz ilişkisini”" veya "iki kanallı gerçek dünya sinyallerinin kanallar arasındaki ilişkisini” dikkate almak için de kullanılabilir. Örneğin; rüzgar sinyallerinin işlenmesinde; rüzgarın genlik ve yön bilgisi kullanarak kompleks-değerli olarak elde edilmesi söz konusu olabilmektedir (Goh vd., 2006) ve benzer yolla MRI sinyalleri de kompleks düzlemde incelebilmektedir (Xu vd., 2008). Kompleks düzlemde faz ve genlik bilgisi anlık olarak da modellenebilmektedir. Bu da gerçek zamanlı akan sinyallerin hem genliğinin hem de fazının anlık olarak tespit edilmesine olanak sağlar (Mandic ve Goh, 2009). Ayrıca, iki reel-değerli sinyali ayrı ayrı işlemek yerine, kompleks düzlemde reel ve imajiner olarak ifade ederek sinyallerin aralarındaki istatistiksel ilişkiler dikkate alınarak tek bir kompleks formda iki sinyal aynı anda işlenebilmektedir (Mandic ve Goh, 2009; Adali ve Haykin, 2010). Sonuç olarak, sinyalin kompleks-değerli olarak temsil edilmesi; gerçek dünya süreçlerinin analizi için matematiksel olarak daha izlenebilir bir çerçeve sağlamaktadır.

Kompleks-değerli sinyaller genellikle düzenli/ikincidereceden dairesel (proper/second-order circular) veya dairesel (circular) kabul edilmektedir. Düzenli kompleks-değerli sinyal tipleri, kendilerinin kompleks eşlenikleri ile ilintisizken, dairesel kompleks-değerli sinyaler, kompleks düzlemde herhangi bir rotasyon durumunda değişmeyen olasılık yoğunluk fonksiyonuna (probability density function, PDF) sahiptirler (Mandic ve Goh, 2009; Adali ve Haykin, 2010; Xia vd., Jelfs vd., 2012; Adali vd., 2011; Schreier ve Scharf, 2010; Mengüç ve Acır, 2017; Mengüç ve Acır, 2018a). Bu varsayımlar, kompleks-değerli sinyallerin hesaplamalarını basite indirgemiş ve reel-değerli versiyonlarına benzer şekilde kompleks-değerli sinyallerin ele alınmasını sağlamıştır. Bilindiği üzere geleneksel kompleks-değerli adaptif filtreleme algoritmaları, genelde kendilerinin reel-değerli versiyonlarının basit uzantılarıdır (Mandic ve Goh, 2009; Adali ve Haykin, 2010; Xia vd., Jelfs vd., 2012; Adali vd., 2011; Schreier ve Scharf, 2010; Mengüç ve Acır, 2017; Mengüç ve Acır, 2018a). Bu kapsamda, ilk olarak Widrow ve arkadaşları (Widrow vd., 1975); kesin lineer (strictly linear, SL) tabanlı olan geleneksel kompleks-değerli LMS (Complex-valued LMS, CLMS) algoritmasını 1975 yılında önermişlerdir. Bu algoritma klasik LMS algoritmasının reel düzlemden kompleks düzleme genişletilmesi şeklinde olup, kompleks-değerli adaptif filtreleme için geliştirilen ilk algoritmadır. CLMS algoritması, sinyallere ait ikinci-dereceden istatistiksel bilgi olarak kovaryans matrisi $\mathbf{C}_{\mathbf{x x}}=E\left\{\mathbf{x x}^{H}\right\}$ bilgisi kullandığından sadece dairesel veriler için 
optimal çözüm sunar (Mandic ve Goh, 2009; Jelfs vd., Khalili vd., 2014; Picinbono ve Chevalier, 1995), burada $\mathbf{x}$ sifir ortalamal kompleks-değerli rastgele vektörü, $H$ eşlenik transpozu, $E\{\bullet\}$ ise beklenen değeri temsil eder. Fakat daha önce bahsedildiği gibi gerçek dünya uygulamalarının çoğunda, düzensiz/ikinci dereceden dairesel olmayan kompleks-değerli veriler ile karşılaşılmaktadır (Mandic ve Goh, 2009; Adali ve Haykin, 2010; Xia vd., Jelfs vd., 2012; Adali vd., 2011; Schreier ve Scharf, 2010; Mengüç ve Acır, 2017; Mengüç ve Acır, 2018a). Bu nedenle, kompleks düzlemde adaptif filtreleme algoritmalarının tasarımları yapılırken; kendisine ait kompleks eşleniği ile ilintisi olan ve PDF'nin kompleks düzlemde rotasyon altında değiştiği kompleks-değerli dairesel olmayan sinyallerin de ayrıca dikkate alınması gerekmektedir (Mandic ve Goh, 2009; Adali ve Haykin, 2010; Xia vd., Jelfs vd., 2012; Adali vd., 2011; Schreier ve Scharf, 2010; Mengüç ve Acır, 2017; Mengüç ve Acır, 2018a). CLMS algoritması gibi geleneksel algoritmalar, yapılarında ikincidereceden istatistiklerin tamamını barındırmadıklarından kompleks-değerli dairesel olmayan sinyallerin işlenmesi için uygun değildir.

Son zamanlarda, geleneksel CLMS algoritmasının bahsi geçen dezavantajlarını elimine eden artırılmış istatistikler, kompleks-değerli adaptif filtreleme alanına ciddi katkılar sunmuştur. Aslında artırılmış istatistikler; bir kompleks-değerli rastgele $\mathbf{x}$ vektörüne ait ikinci dereceden istatistiksel özelliklerin tamamının elde edilebilmesi için sadece kovaryans matrisinin $\mathbf{C}_{\mathbf{x x}}=E\left\{\mathbf{x x}^{H}\right\}$ kullanımının yeterli olmadığını, dairesel olmayan sinyallerin de analiz edilebilmesi için sözde-kovaryans $\mathbf{P}_{\mathbf{x x}}=E\left\{\mathbf{x x}^{T}\right\}$ matrisinin de hesaba katılması gerektiğini ortaya koymuştur. Bu kapsamda, ilk olarak kompleks-değerli rastgele $\mathbf{x}$ vektörüne ait artırılmış kompleks-değerli vektör $\mathbf{x}^{a}=\left[\mathbf{x}^{T}, \mathbf{x}^{H}\right]^{T}$

tanımlanmış ve ardından ise $\mathbf{x}$ 'e ait hem kovaryans hem de sözde kovaryans matrisleri (1)'deki gibi sunulan tek bir artırılmış kovaryans matrisinin içerisine yerleştirilmiştir (Mandic ve Goh, 2009; Adali ve Haykin, 2010; Xia vd., Jelfs vd., 2012; Adali vd., 2011; Schreier ve Scharf, 2010; Mengüç ve Acır, 2017; Mengüç ve Ac1r, 2018a) :

$\mathbf{C}_{\mathbf{x}^{a} \mathbf{x}^{a}}=\left[\begin{array}{ll}\mathbf{C}_{\mathbf{x x}} & \mathbf{P}_{\mathbf{x x}} \\ \mathbf{P}_{\mathbf{x x}}^{*} & \mathbf{C}_{\mathbf{x x}}^{*}\end{array}\right]$

burada $\mathbf{P}_{\mathbf{x x}}=\mathbf{0}$ olması durumunda; $\mathbf{x}$ vektörü, düzenlidir yani ikinci-dereceden daireseldir. Aksi halde $\mathbf{x}$ vektörü düzensizdir yani ikinci-dereceden dairesel değildir. Sonuç olarak, artırılmış kovaryans matrisi $\mathbf{C}_{\mathbf{x}^{a} \mathbf{x}^{a}}$; hem "kovaryans" hem de "sözde kovaryans" matrisi bilgisi içerdiğinden dolayı, dairesel ve dairesel olmayan kompleks-değerli sinyallerin ikinci-dereceden istatistiksel özelliklerinin tamamını kapsamaktadır (Mandic ve Goh, 2009). Böylece, artırılmış istatistikler; WL modele dayanan, artırılmış kompleks-değerli en küçük ortalama kare (Augmented CLMS, ACLMS) (Javidi vd., 2008) algoritmasının tasarımının yolunu açmış ve adaptif filtreleme tekniklerine entegre edilmesi; dairesel olmayan kompleks-değerli sinyallerin yer aldığ uygulamalarda başarımı önemli ölçüde arttırmıştır. Bu sayede, hem dairesel hem de dairesel olmayan sinyallerin işlenmesine olanak sağlanmıştır.

$\mathrm{Bu}$ çalışmada ise, Parkinson hastalarında sıklıkla görülen patolojik dinlenme tremorlerinin kompleks düzlemde adaptif tahmini gerçekleştirilmiştir. $\mathrm{Bu}$ amaç doğrultusunda, öncelikle anlık olarak ölçülen patolojik "sağ el ve sol el" veya "sağ bacak ve sol bacak" tremorleri kompleks düzlemde tek bir formda ifade edilmiştir. Ardından ise bu patolojik tremorler, bir-adım-ileri SL ve WL tabanlı tahmin ediciler kullanılarak adaptif olarak tahmin edilmiştir. Tahmin işlemlerinde kullanılan SL tabanlı tahmin edici geleneksel CLMS algoritması ile eğitilirken, WL tabanlı tahmin edici ise son zamanlarda önerilen ACLMS algoritması ile eğitilmiştir. SL ve WL tabanlı tahmin edicilerin başarımları, ise gerçek dünya verisi olan patolojik dinlenme tremorleri üzerinde mutlak hata ve tahmin kazancı açısından detaylı bir şekilde incelenmiştir. Yapılan benzetim sonuçları; kompleks-değerli patolojik dinlenme tremorlerinin dairesel olmayan davranış sergilediğini ve bu yüzden de WL tabanlı tahmin edicinin, SL versiyonuna kıyasla daha üstün bir başarım sergilediğini ortaya koymuştur.

\section{Materyal ve Metot}

$\mathrm{Bu}$ bölümde, tremor sinyallerinin tahmininde kullanılan CLMS ve ACLMS algoritmalarından sırasıyla bahsedilmiştir.

\subsection{CLMS Algoritması}

CLMS algoritması aslında LMS algoritmasının kompleks düzlemde çalışan versiyonudur. CLMS algoritması, filtre çıkış sinyalini hesaplarken SL model yapısını kullandığından SL algoritmalar ailesine aittir. CLMS algoritması, sadece kompleksdeğerli dairesel sinyaller için optimum çözüm sunar ve dairesel olmayan sinyallerde beklenen başarımı sergileyemez. $\mathrm{Bu}$ durumun temel sebebi CLMS algoritmasının, ikinci-dereceden istatistiksel bilgi olarak sadece kovaryans matrisi bilgisini kullanmasıdır.

CLMS algoritması, bir sonlu dürtü cevaplı (finite impulse response, FIR) filtrenin ağırlık vektörünü güncellemek için anlık hata sinyali $e_{n}$ kullanılarak tanımlanan maliyet fonksiyonu $J_{n}$ 'ni minimize etmeyi amaçlar:

$$
J_{n}=\frac{1}{2} E\left\{\left|e_{n}\right|^{2}\right\}=\frac{1}{2} E\left\{e_{n} e_{n}^{*}\right\}
$$

burada anlık hata sinyali $e_{n}$, beklenen sinyal $d_{n}$ ile SL model tabanlı FIR filtre çıkış sinyali $y_{n}$ arasındaki farkı temsil eder:

$$
e_{n}=d_{n}-y_{n}=d_{n}-\mathbf{h}_{n}^{H} \mathbf{x}_{n} .
$$

Denklem (3)'de yer alan $\mathbf{h}_{n} \in \mathbb{C}^{N x 1}$ ifadesi, içerisinde ağırlık katsayılarının olduğu bir ağırlı vektörünü, $\mathbf{x}_{n}=\left[x_{n}, \ldots, x_{n-N+1}\right] \in \mathbb{C}^{N x 1}$ ise filtre giriş sinyalini temsil etmektedir. Ayrıca $N$ ifadesi, SL tabanlı FIR filtrenin derecesidir.

Deklem (2)'de yer alan maliyet fonksiyonu $J_{n}$ 'nin, ağırlık vektörü $\mathbf{h}_{n} \in \mathbb{C}^{N x 1}$ 'e göre minimizasyonunun sonucunda; CLMS algoritmasına ait ağırlık vektörü güncelleme kuralı aşağıdaki formda elde edilir:

$\mathbf{h}_{n+1}=\mathbf{h}_{n}+\mu e_{n}^{*} \mathbf{x}_{n}$

burada $\mu$ CLMS algoritmasının adım büyüklüğünü temsil etmektedir.

Son olarak algoritmanın sözde kodu adım adım Tablo 1'de sunulmuştur. Ayrıca, CLMS algoritmasının türetilmesine ait 
gerekli bilgiler, (Widrow vd.,1975, Mandic ve Goh, 2009)'da yer alan çalışmada mevcuttur.

Tablo 1. CLMS Algoritmasının Sözde Kodu

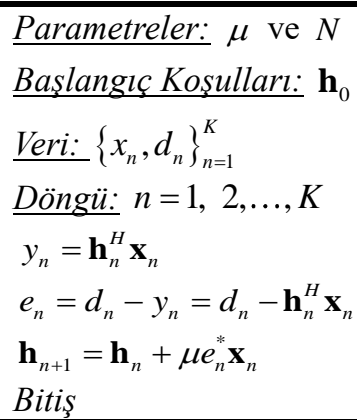

\subsection{ACLMS Algoritması}

ACLMS algoritması, CLMS algoritması gibi kompleks düzlemde çalışma kabiliyetine sahip bir algoritmadır. CLMS'nin aksine, ACLMS algoritması filtre çıkış sinyalini hesaplarken WL model yapısını kullanır ve WL algoritmalar ailesine ait önemli bir adaptif filtreleme algoritmasıdır. WL model yapısının kullanımı, ACLMS algoritmasına hem dairesel hem de dairesel olmayan kompleks-değerli sinyallerin işlenmesi için optimal sonuç sunmasını sağlar. ACLMS algoritması, CLMS algoritmasına benzer bir şekilde denklem 3'de yer alan maliyet fonksiyonu $J_{n}$ 'ni minimize etmeyi amaçlar ancak; bu algoritmanın temel farkı, $J_{n}$ tanımlanırken anlık hata sinyali $e_{n}$ 'nin denklem (6)' da ki gibi WL tabanlı bir FIR filtre çıkış sinyali $y_{n}$ kullanılarak ifade edilmesidir:

$J_{n}=\frac{1}{2} E\left\{\left|e_{n}\right|^{2}\right\}=\frac{1}{2} E\left\{e_{n} e_{n}^{*}\right\}$

$e_{n}=d_{n}-y_{n}=d_{n}-\mathbf{h}_{n}^{H} \mathbf{x}_{n}-\mathbf{g}_{n}^{H} \mathbf{x}_{n}^{*}$

burada $\mathbf{h}_{n} \in \mathbb{C}^{N x 1}$ ve $\mathbf{g}_{n} \in \mathbb{C}^{N x 1}$ sirasiyla WL tabanlı FIR filtrenin standart ve eşlenik ağırlık vektörlerini temsil etmektedir.

Denklem (5)'in standart $\mathbf{h}_{n} \in \mathbb{C}^{N x 1}$ ve eşlenik $\mathbf{g}_{n} \in \mathbb{C}^{N x 1}$ ağırlık vektörlerine göre minimizasyonun sonucunda ise ACLMS algoritmasına ait standart ve eşlenik ağırlık vektörü güncelleme kuralları aşağıdaki formda elde edilir:

$\mathbf{h}_{n+1}=\mathbf{h}_{n}+\mu e_{n}^{*} \mathbf{x}_{n}$

$\mathbf{g}_{n+1}=\mathbf{g}_{n}+\mu e_{n}^{*} \mathbf{x}_{n}^{*}$
Son olarak ise $\mathbf{h}_{n} \in \mathbb{C}^{N x 1}$ ve $\mathbf{g}_{n} \in \mathbb{C}^{N x 1}$ bir artırılmış ağırlık vektörü $\quad \mathbf{w}_{n}^{a} \in \mathbb{C}^{2 N \times 1}$ içerisine yerleştirildiğinde ACLMS algoritmasının artırılmış ağırlık vektörüne ait güncelleme kuralı aşağıdaki gibi elde edilir.

$\mathbf{w}_{n+1}^{a}=\mathbf{w}_{n}^{a}+\mu e_{n}^{*} \mathbf{x}_{n}^{a}$

burada $\mathbf{x}^{a}=\left[\mathbf{x}^{T}, \mathbf{x}^{H}\right]^{T} \in \mathbb{C}^{2 N \times 1}$ artırılmış giriş vektörünü temsil etmektedir. Algoritmanın sözde kodu Tablo 2'de detaylı bir şekilde sunulmuştur. Ayrıca, ACLMS algoritmasının türetilmesine ait detaylı bilgilere için (Mandic ve Goh, 2009)'de yer alan çalışmadan ulaşılabilir.

Tablo 2. ACLMS Algoritmasinin Sözde Kodu

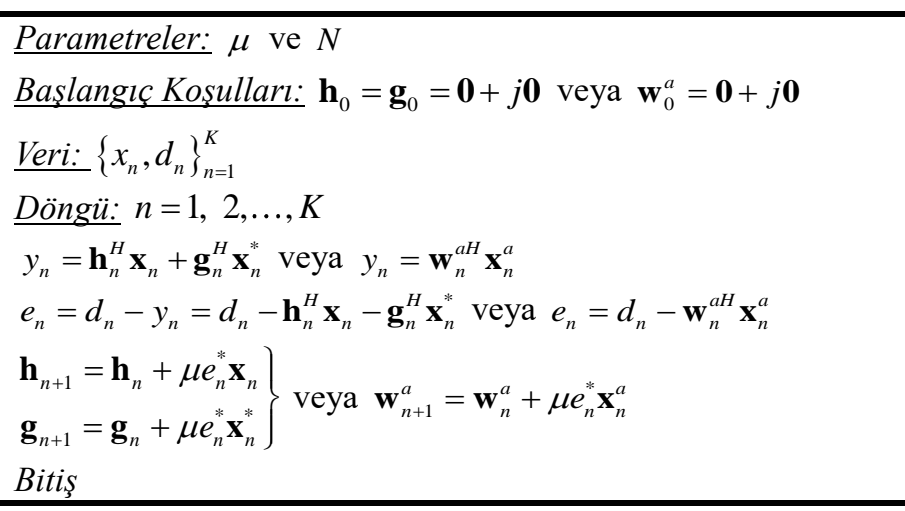

\subsection{Patolojik Dinlenme Tremorleri için Önerilen Bir-Adım- İleri Tahmin Ediciler}

Bu bölümde, patolojik dinlenme tremorleri için önerilen bir-adımileri tahmin edicilerin çalışma prensiplerinden bahsedilmiştir. Burada, iki farklı kanaldan ölçülen tremor sinyallerini ayrı ayrı tahmin etmek yerine kompleks düzlemde reel ve imajiner bileşenler olarak temsil edilerek tek bir formda anlık olarak tahmin edilmesi amaçlanmıştır. Şekil 1 (a) ve (b)'de sırasıyla patolojik dinlenme tremorleri için SL ve WL tabanlı kompleks düzlemde önerilen bir-adım-ileri tahmin edicilere ait blok diyagramlara yer verilmiştir. Şekil 1'den görüldüğü üzere "sağ el $\left(T_{R H, n}\right)$ ve sol el $\left(T_{L H, n}\right)$ " veya "sağ bacak $\left(T_{R L, n}\right)$ ve sol bacak ( $T_{L L, n}$ )"tan alınan patolojik dinlenme tremorleri öncelikle reel ve imajiner bileşenlerin toplamı olacak şekilde kompleks düzlemde ifade edilmiştir. Aslında bu ifadeler, tahmin edicilerin beklenen sinyali $d_{n}$ 'ni temsil etmektedir.

$d_{n}=T_{R H, n}+j T_{L H, n}$

$d_{n}=T_{R L, n}+j T_{L L, n}$ 


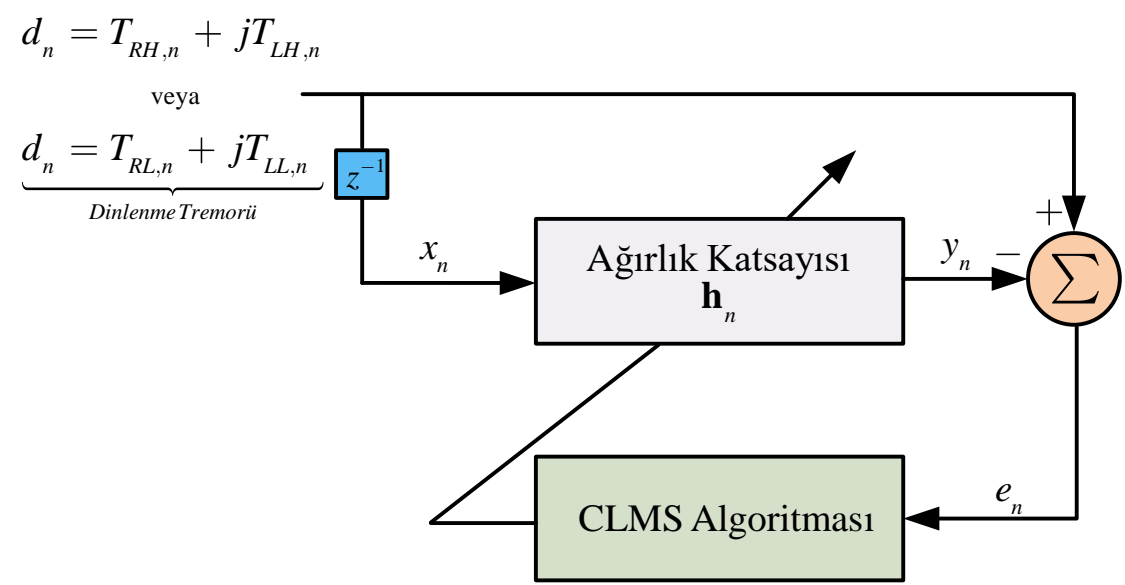

(a)

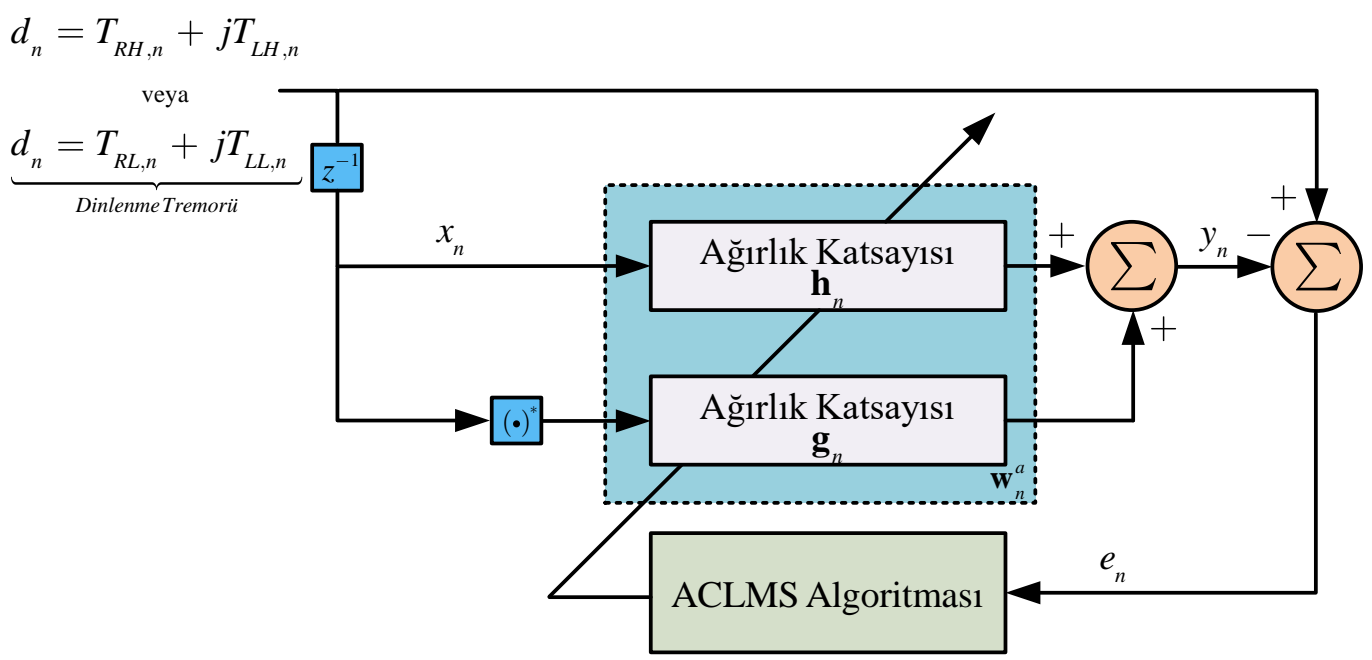

(b)

Şekil 1. Patolojik Dinlenme Tremorleri için Kompleks Düzlemde Önerilen Bir-Adım-İleri Tahmin Ediciler (a) SL Tabanlı Tahmin Edici (b) WL Tabanlı Tahmin Edici

Tahmin modunda çalışılmasından dolayı, giriş sinyali $x_{n}$, beklenen sinyal $d_{n}$ 'nin bir birim gecikmiş halidir, yani $x_{n}=d_{n-1}$ 'dir. Ayrıca, Şekil 1 (a)'daki SL tabanlı tahmin edicinin ağırlık vektörü CLMS algoritması ile güncellenirken, Şekil 1 (b)'deki WL tabanlı tahmin edicinin ağırlık vektörleri ACLMS algoritması yardımıyla güncellenmektedir.

Sonuç olarak, iki kanaldan alınan reel-değerli patolojik tremor sinyalleri kompleks düzlemde ifade edilerek, kompleks düzlemin sunmuş olduğu hem cebirsel hem de istatistiksel avantajlardan faydalanılmış ve kanallar arasındaki ilişki dikkate alınmıştır.

\section{Araştırma Sonuçları ve Tartışma}

\subsection{Patolojik Dinlenme Tremorüne ait Veri Kümesi}

Bu çalışma kapsamında, literatürde yaygın kullanılan "Motus Biyomühendislik A.Ş.” (Motus Bioengineering Inc.) sunduğu veri setinde yer alan "Chprob3b" olarak isimilendirilen aynı hastaya ait ve ölçülen sağ el, sol el, sağ bacak ve sol bacaktan aynı anda ölçülerek kaydedilen 4 adet patolojik dinlenme tremorü kullanılmıştır. İlgili ölçümler $100 \mathrm{~Hz}$ örnekleme frekansına sahip 10 saniyelik tek eksenli tremor işaretleridir (Reeke, 2005).
Şekil 2'de bu tremor sinyallerinin her birine yer verilmiştir. Ayrıca, Şekil 3 'te kompleks düzlemde ifade edilen patolojik el ve bacak dinlenme tremorlerinin geometrik görünümü sunulmuştur. Şekil 3'ten de görüldüğü üzere kompleks düzlemde ifade edilen patolojik el ve bacak dinlenme tremorleri dairesel olmayan davranış sergilemiştir. Bu durumda, artırılmış istatistiklerin; yani, WL tabanlı modellemenin, tahmin başarımını ciddi oranda artırabileceği beklenmektedir. Aynı zamanda Şekil 3'ü daha da desteklemek için her iki kompleks-değerli sinyal için dairesellik dereceleri, (Ollila, 2008)'de yer alan metod yardımıyla ölçülmüş ve el tremorü için dairesellik indeksi 0.4748 , bacak tremorü için ise 0.1716 olarak bulunmuştur. Bu değerlerdeki, bahsi geçen iki tremor sinyalinin dairesel olmadığını ayrıca göstermiştir.

\subsection{SL ve WL Tabanlı Tahmin Edicilerin Parametre Seçimi}

Adil bir karşılaştırma için hem SL hem de WL tabanlı tahmin edicilerde kullanılan FIR filterenin derecesi $N=32$ olarak seçilmiştir. Tahmin edicilerin ağırlık vektörlerini güncelleyen CLMS ve ACLMS algoritmalarının adım büyüklüğü değeri ise $\mu=0.01$ 'e kurulmuştur.

\subsection{Benzetim Sonuçları ve Tartışma}

Şekil 4 (a) ve (b)'de sırasıyla kompleks düzlemde ifade edilen patolojik el ve bacak dinlenme tremorleri için bir-adım-ileri SL 
(CLMS) ve WL (ACLMS) tabanlı tahmin edicilerin mutlak hatalarına yer verilmiştir. Şekil 4'den görüldüğü üzere, her iki tremor için de ACLMS algoritması ile eğitilmiş WL tabanlı tahmin edici, CLMS ile eğitilmiş olan SL tabanlı tahmin ediciden mutlak hata anlamında daha iyi bir tahmin başarımı sağlamıştır. Aslında bu başarım beklenen bir durumdur. Çünkü her iki tremor sinyali; hem reel-imajiner eksendeki geometrik görünümde hem de dairesellik indeksi ölçümünde dairesel olmayan davranış sergilemiştir. Sonuç olarak WL yapı tabanlı artırılmış istatistikler, tahmin başarımlarını ciddi manada artırmıştır. Bu grafiksel sonuçları daha da desteklemek amaciyla Tablo 3'de, hem SL hem de WL tabanlı tahmin edicilerin tahmin kazanci $R_{p}(\mathrm{~dB})$ başarımlarına yer verilmiştir. $R_{p}$ ifadesi ise aşağıda verilen denklem kullanılarak hesaplanmıştır (Mengüç ve Acır, 2018):

$$
R_{p}=10 \log _{10} \sigma_{d}^{2} / \sigma_{e}^{2}[d B]
$$

burada $\sigma_{d}^{2}$ ve $\sigma_{e}^{2}$ sirasiyla beklenen ve hata sinyallerinin varyansını temsil etmektedir. Tablo 3'den de anlaşıldığ iyi tahmin kazancı değerini, WL tabanlı tahmin edici sağlamıştır.

Sonuç olarak, yapılan bu çalışma ile iki kanaldan alınan reeldeğerli patolojik tremorler kompleks düzlemde ifade edilerek, kompleks düzlemin sunmuş olduğu hem cebiresel hem de istatistiksel avantajlardan faydalanılmış ve kanallar arasındaki ilişki dikkate alınmıştır.

Tablo 3. Kompleks-Değerli Patolojik Tremorler için SL ve WL Tabanlı Tahmin Edicilerin Tahmin Kazancı (dB) Başarımları

\begin{tabular}{c|c|c}
\hline \multirow{2}{*}{$\begin{array}{c}\text { Kompleks-Değerli } \\
\text { Patolojik Tremorleri }\end{array}$} & \multicolumn{2}{c}{ Tahmin Edicilerin $R_{p}$} \\
\cline { 2 - 3 } & (dB) Tahmin Kazançları \\
\cline { 2 - 3 } & $\begin{array}{c}\text { SL } \\
\text { (CLMS) }\end{array}$ & $\begin{array}{c}\text { WL } \\
\text { (ACLMS) }\end{array}$ \\
\hline El & 13.8147 & 15.1900 \\
\hline Bacak & 10.8325 & 11.4996 \\
\hline
\end{tabular}

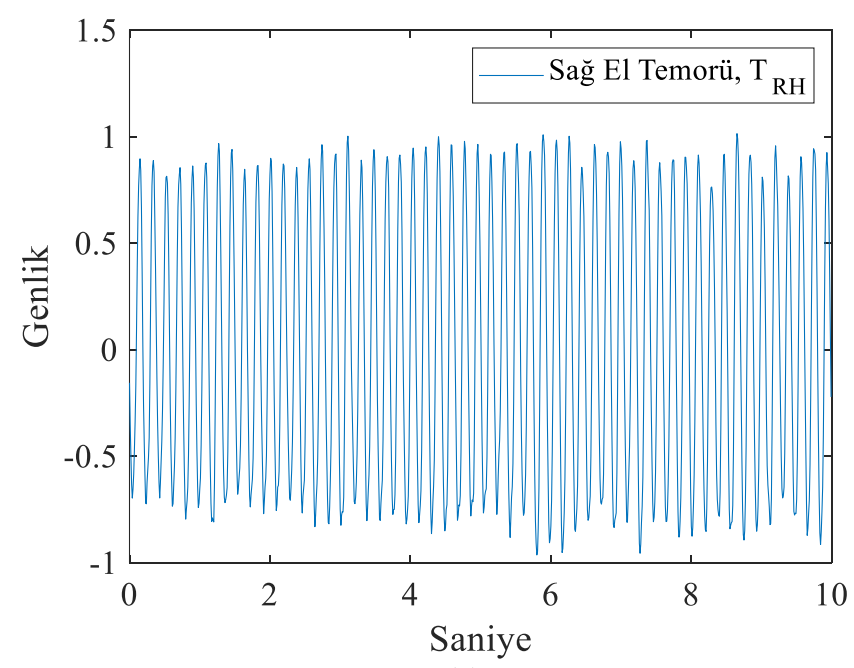

(a)

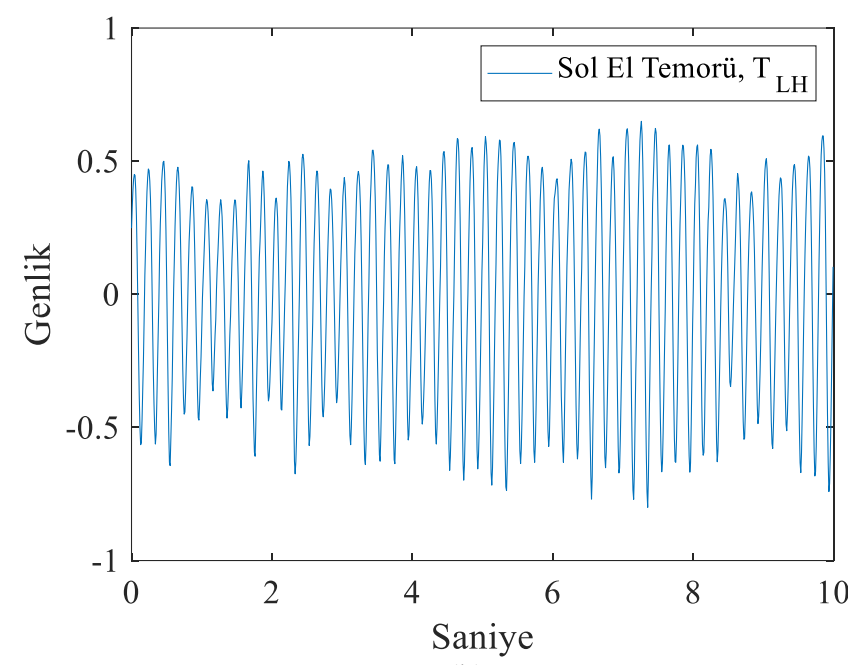

(b)

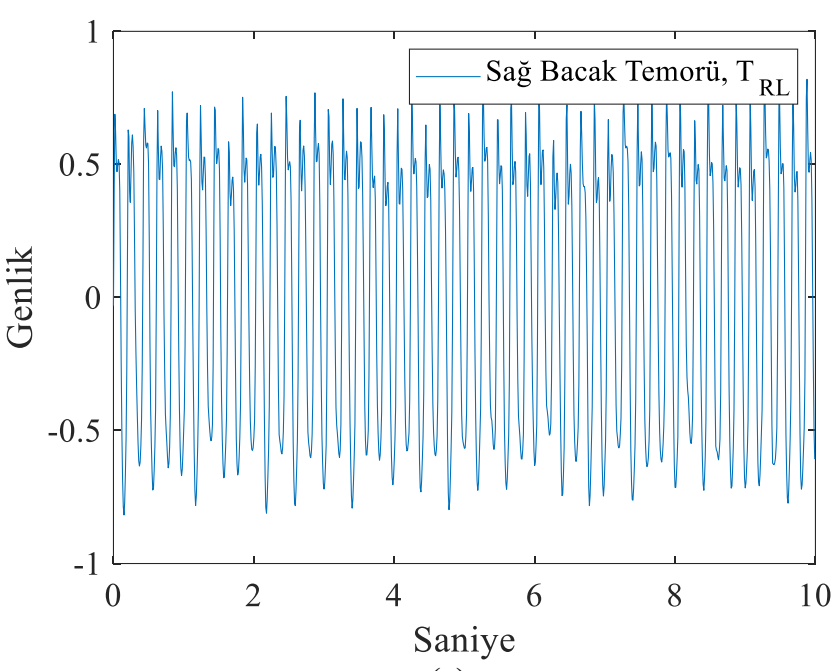

(c)

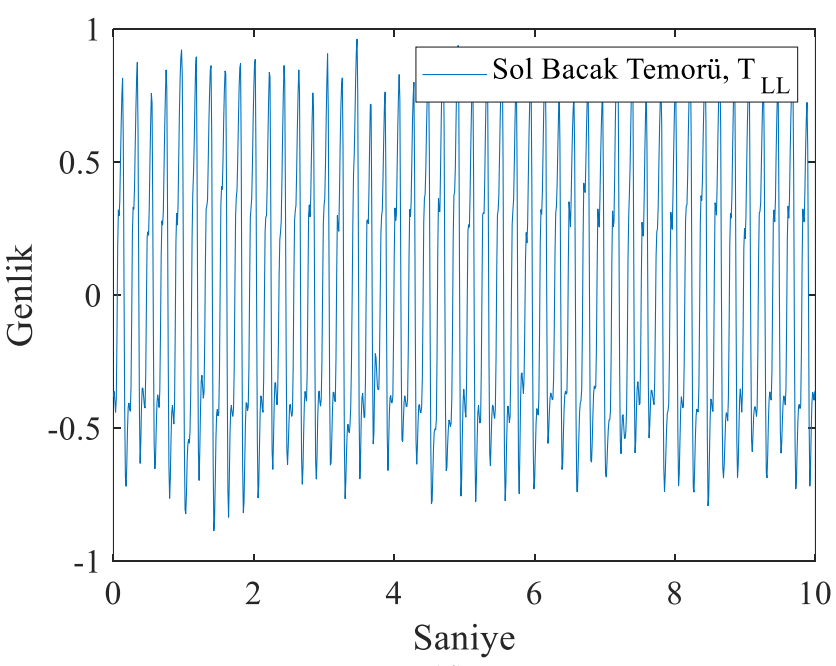

(d)

Şekil 2. Patolojik Dinlenmede Tremorleri (a) Să̆ El (b) Sol El (c) Să̆ Bacak (d) Sol Bacak 


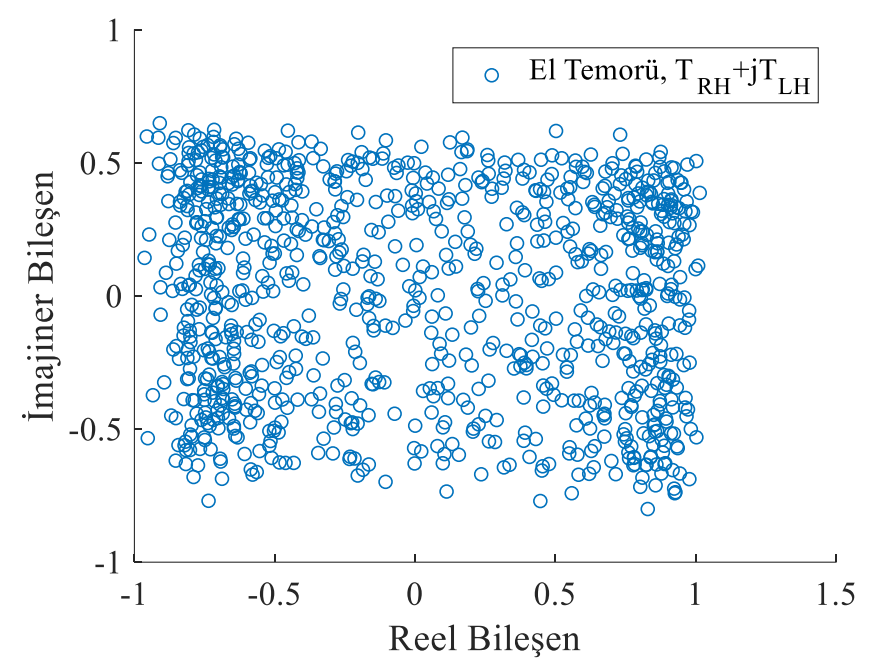

(a)

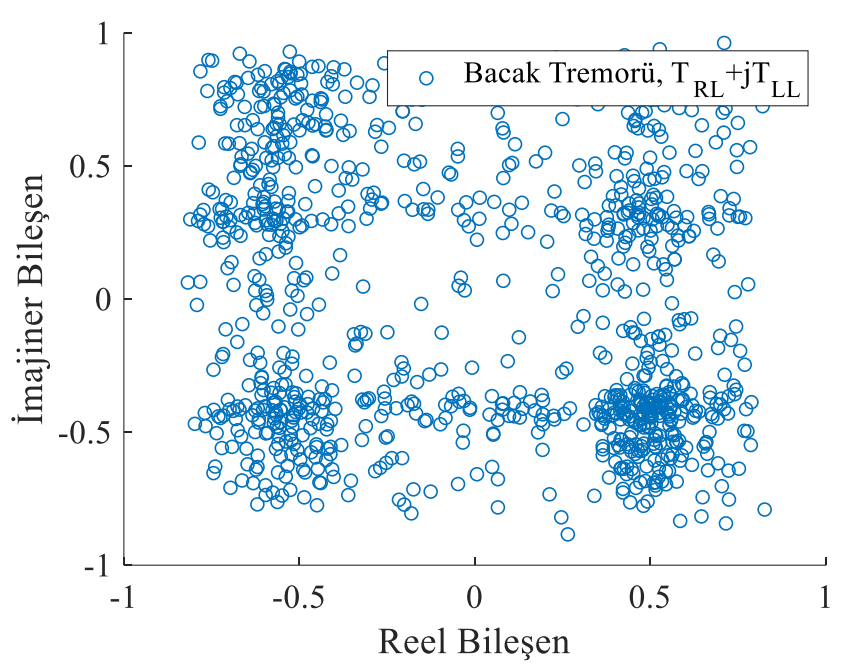

(b)

Şekil 3. Kompleks Düzlemde Patolojik Dinlenme Tremorlerinin Geometrik Görünümü (a) El (b) Bacak

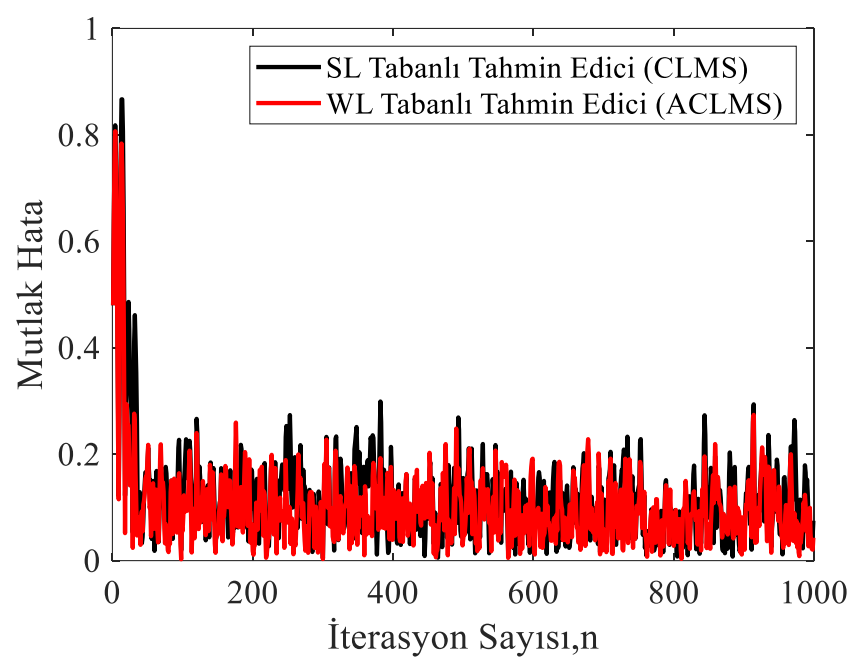

(a)

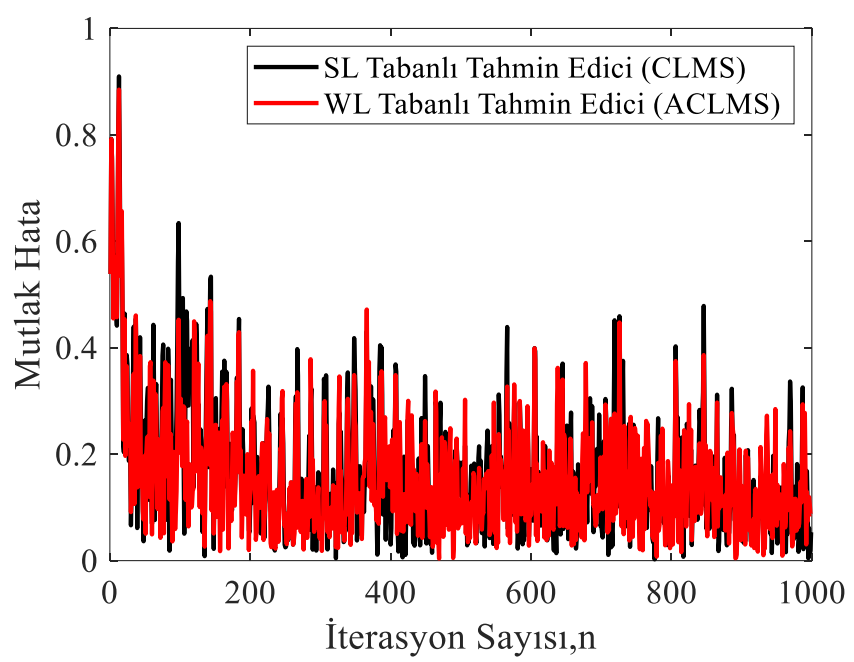

(b)

Şekil 4. Bir-Adım-İleri SL (CLMS) ve WL (ACLMS) Tabanlı Tahmin Edicilerin Mutlak Hatalarl (a) Patolojik El Tremorü,

$$
d_{n}=T_{R H, n}+j T_{L H, n} \text { (b) Patolojik Bacak Tremörü, } d_{n}=T_{R L, n}+j T_{L L, n}
$$

\section{Sonuç}

$\mathrm{Bu}$ çalışmada, Parkinson hastalarında sıklıkla görülen patolojik dinlenme tremorleri kompleks düzlemde adaptif olarak tahmin edilmiştir. Bu amaçla, ilk olarak anlık olarak ölçülen patolojik "sağ el ve sol el" veya "sağ bacak ve sol bacak" tremorleri kompleks düzlemde ifade edilmiş ve ardından biradım-ileri SL ve WL tabanlı tahmin ediciler kullanılarak adaptif olarak tahmin edilmiştir. Burada, SL tabanlı tahmin edici geleneksel CLMS algoritması ile eğitilirken, WL tabanlı tahmin edici ise son zamanlarda önerilen ACLMS algoritması ile eğitilmiştir. SL ve WL tabanlı tahmin edicilerin başarımları, ise gerçek dünya verisi olan patolojik el ve bacak dinlenme tremorleri üzerinde mutlak hata ve tahmin kazancı açısından detaylı bir şekilde incelenmiştir. Yapılan benzetim sonuçları; kompleksdeğerli patolojik dinlenme tremorlerinin dairesel olmayan davranış sergilediğini ve bu nedenle WL tabanlı tahmin edicinin, SL versiyonuna kıyasla daha üstün bir başarım sunduğunu ortaya koymuştur. Sonuç olarak, iki kanaldan alınan reel-değerli patolojik tremorler kompleks düzlemde ifade edilerek, kompleks düzlemin sunmuş olduğu hem cebiresel hem de istatistiksel avantajlardan faydalanılmış ve kanallar arasındaki ilişki dikkate alınmıştır.

\section{Kaynakça}

Adali ,T., Haykin, S. 2010. Adaptive Signal Processing: Next Generation Solutions, Wiley: IEEE Press.

Adali, T., Calhoun, V. D.2007. "Complex ICA of medical imaging data”, IEEE Signal Proc. Mag., 24(5),136-139.

Adali, T., Schreier, P. J., Scharf,L. L. 2011. "Complex-valued signal processing: The proper way to deal with impropriety" , IEEE Trans. Signal Process., 59(11), 5101-5125.

Atashzar, S.F., Shahbazi, M., Samotus, O., Tavakoli, M., Jog, M.S. and Patel, R.V. (2016) . Characterization of Upper-Limb Pathological Tremors: Application to Design of An Augmented Haptic Rehabilitation System. IEEE J. Sel. Topics Signal Process., 10(5), pp. 888-903. 
Elble, R. and Koller, W. (1990). Tremor, Johns Hopkins University Press, Baltimore,USA, (MD thesis).

Elble, R.J. (1997). The pathophysiology of tremor, in: R.L. Watts, W.C. Koller (Eds.), Movement Disorders: Neurologic Principles and Practice,McGraw-Hill, New York, pp. 405417.

Goh, S. L., Chen, M. , Popovic,D. H., Aihara, K. , Obradovic, D., Mandic, D. P. 2006. "Complex-valued forecasting of wind profile," Renewable Energy, 31, 1733-1750.

Javidi,S., Goh,S. L., Pedzisz, M., Mandic,D. P. 2008. "The augmented complex least mean square algorithm with application to adaptive prediction problems", in Proc. 1st IARP Workshop Cogn. Inform. Process., 54-57.

Jelfs,B., Mandic, D. P., Douglas, S. C. 2012. "An adaptive approach for the identification of improper complex signals," Signal Process., 92, 335-344.

Khalili, A., Rastegarnia, A., Bazzi, W. M., Yang,Z. 2014. "Derivation and analysis of incremental augmented complex leastmean square algorithm", IET Signal Process., 9(4), 312 319.

Mandic, D. P., Goh, S. L. 2009. Complex Valued Nonlinear Adaptive Filters: Noncircularity Widely Linear and Neural Models. United Kingdom: Wiley.

Maneski, L. P. et al. (2011). Electrical stimulation for the suppression of pathological tremor. Medical and Biological Engineering and Computing, 49(10), pp. 1187-1193.

Mengüç, E. C., Çınar, S., Xiang, M., \& Mandic, D. P. (2021). Online Censoring Based Weighted-Frequency Fourier Linear Combiner for Estimation of Pathological Hand Tremors. IEEE Signal Processing Letters, 28, 1460-1464.

Mengüç, E.C ve Rezayi, N. (2019). Estimation of Pathological Hand Signals by Fourier Linear Combiner Based Online Censoring LMS Algorithm, in: IEEE 27.Sinyal İşleme ve İletişim Uygulamaları Kurultayı. Sivas. pp. 1-4.

Mengüç, E.C., Acır, N. 2017. "An augmented complex-valued Lyapunov stability theory based adaptive filter algorithm," Signal Processing., 137, 10-21.

Mengüç, E.C., Acır, N. 2018. “An augmented complex-valued least-mean kurtosis algorithm for the filtering of noncircular signals," IEEE Transactions on Signal Processing, 66(2), 438-448.

O'Connor, R. J. and Kini, M. U. (2011). Non-pharmacological and nonsurgical interventions for tremor: a systematic review. Parkinsonism and Related Disorders, 17(7), pp. 509-515.

Ollila, E. (2008). On the circularity of a complex random variable. IEEE Signal Processing Letters, 15, 841-844.

Picinbono, B. , Chevalier, P. 1995. "Widely linear estimation with complex data," IEEE Trans. Signal Process., 43(8),20302033.

Reeke, G. N. 2005. "Modeling in the Neurosciences: From Biological Systems to Neuromimetic Robotics", CRC Press, [online] Available: https://www.motusbioengineering.com/.

Rezayi, N. and Mengüç, E. C. (2018) Performances of LMSbased adaptive Fourier linear combiners on the estimation of pathological hand tremors. in: National Conf. on Electr. Electron. and Biomed. Engineering, pp. 543-547.

Riviere, C. N. and Thakor, N. V. (1996). Modeling and canceling tremor in human-machine interfaces. in: IEEE Eng. Med., Biol. Mag., 15(3), pp. 29-36.

Riviere, C. N., Rader, R. S. and Thakor, N. V. (1998). Adaptive cancelling of physiological tremor for improved precision in microsurgery. in: IEEE Trans. Biomed. Engineering, 45(7), pp. 839-846.

Riviere, C.N., Ang, W.T. and Khosla, P.K. (2003). Toward active tremor canceling in handheld microsurgical instruments. IEEE Trans. Robot. Automat. 19(5), pp. 793-800.

Riviere,C. N., Reich, S. G. and Thakor, N. V. (1997)Adaptive fourier modeling for quantification of tremor1. Journal of Neuroscience Methods, vol. 74, no. 1, pp. 77-87.

Rocon, E., Belda-Lois, J., Ruiz, A., Manto, M., Moreno, J. C. and Pons, J. (2007a). Design and validation of a rehabilitation robotic exoskeleton for tremor assessment and suppression. in: IEEE Transactions on Neural Systems and Rehabilitation Engineering, 15(3), pp. 367-378.

Rocon, E., Manto, M., Pons, J., Camut, S. and Belda, J. M. (2007b). Mechanical suppression of essential tremor. The Cerebellum, 6(1), pp. 73-78.

Schreier ,P. J., Scharf, L. L. 2010. Statistical Signal Processing of Complex- Valued Data: The Theory of Improper and Noncircular Signals (1.Basim). Cambridge, U.K.: Cambridge Univ. Press.

Stott, A., Kanna, S., Mandic, D. P. 2018. "Widely linear complex partial least squares for latent subspace regression", Signal Processing, 152, 350-362.

Vaz, C., Kong, X. and Thakor, N. (1994). An adaptive estimation of periodic signals using a Fourier linear combiner. in: IEEE Trans. Signal Process., 42(1), pp. 1-10.

Veluvolu, K. and Ang, W. (2010). Estimation and filtering of physiological tremor for real-time compensation in surgical robotics applications. The International Journal of Medical Robotics and Computer Assisted Surgery, 6(3), pp. 334-342.

Veluvolu, K. C., Tan, U.-X., Latt, W. T., Shee, C. and Ang, W. T. (2007). Bandlimited multiple fourier linear combiner for realtime tremor compensation. in: IEEE Annual Engineering in Medicine and Biology Society (EMBS) Conference, pp. 2847-2850.

Veluvolu, K., Latt, W. and Ang, W. (2010). Double adaptive bandlimited multiple fourier linear combiner for real-time estimation/filtering of physiological tremor. Biomedical Signal Processing and Control, 5(1), pp. 37-44.

Wang, S., Gao, Y., Zhao, J. and Cai, H. (2014). Adaptive sliding bandlimited multiple fourier linear combiner for estimation of pathological tremor, Biomedical Signal Processing and Control, 10, pp. 260- 274.

Widrow, B. , McCool, J. , Ball, M. 1975. "The complex LMS algorithm", Proceedings of the IEEE, 63(4), 719-720.

Xia, Y., Douglas, S.C., Mandic, D.P. 2012. “Adaptive frequency estimation in smart grid applications: exploiting 
noncircularity and widely linear adaptive estimators", IEEE Signal Process. 29(5), 44-54.

Xu, L., Pearlson, G. D., Calhoun, V. D. 2008 “Joint source based morphometry to identify sources of gray matter and white matter relative differences in schizophrenia versus healthy controls " In Proc. ISMRM, Toronto. 